\title{
Sleep, Experimental Pain and Clinical Pain in Patients with Chronic Musculoskeletal Pain and Healthy Controls
}

This article was published in the following Dove Press journal: Journal of Pain Research

\author{
Cindy Stroemel-Scheder' \\ Anna Julia Karmann' \\ Elisabeth Ziegler' \\ Michael Heesen ${ }^{2}$ \\ Katrin Knippenberg-Bigge ${ }^{3}$ \\ Philip M Lang ${ }^{4}$ \\ Stefan Lautenbacher (ID) \\ 'Physiological Psychology, University of \\ Bamberg, Bamberg, Germany; \\ ${ }^{2}$ Department of Anesthesiology and Pain \\ Therapy, Kantonsspital Baden, Baden, \\ Switzerland; ${ }^{3}$ Center for Pain Medicine, \\ Regiomed Klinikum Lichtenfels, \\ Lichtenfels, Germany; ${ }^{4}$ Department of \\ Anesthesiology and Pain Therapy, \\ Sozialstiftung Bamberg, Bamberg, \\ Germany
}

Purpose: Everyday variations in night sleep in healthy pain-free subjects are at most weakly associated with pain, whereas strong alterations (eg, sleep deprivation, insomnia) lead to hyperalgesic pain changes. Since it remains unclear how substantial sleep alterations need to be in order to affect the pain system and lead to a coupling of both functions, the present study aimed at providing sufficient variance for co-variance analyses by examining a sample consisting of both healthy subjects and chronic pain patients.

Methods: A sample of 20 chronic musculoskeletal pain patients and 20 healthy controls was examined. This sample was assumed to show high inter-individual variability in sleep and pain, as pain patients frequently report sleep disturbances, whereas healthy subjects were required to be pain-free and normal sleepers. Sleep of two non-consecutive nights was measured using portable polysomnography and questionnaires. Experimental pain parameters (pressure pain thresholds (PPT), temporal summation of pain (TSP), conditioned pain modulation (CPM)) and situational pain catastrophizing (SCQ) were assessed in laboratory sessions before and after sleep. Pain patients' clinical pain was assessed via questionnaire.

Results: As expected, both groups differed in several sleep parameters (reduced total sleep time and sleep efficiency, more time awake after sleep onset, lower subjective sleep quality in the patients) and in a few pain parameters (lower PPTs in the patients). In contrast, no differences were found in TSP, CPM, and SCQ. Contrary to our expectations, regression analyses indicated no prediction of overnight pain changes by sleep parameters.

Conclusion: Since sleep parameters were hardly apt to predict overnight pain changes, this leaves the association of both systems mainly unproven when using between-subject variance for verification.

Keywords: sleep, chronic pain, experimental pain, clinical pain

\section{Introduction}

The effects of sleep on pain are widely known and acknowledged. Sleep deprivation studies suggest profound effects of sleep alterations on pain perception, bodily complaints, and spontaneous pain complaints. ${ }^{1-4}$ This is further underlined by findings of pain processing abnormalities in patients with primary insomnia (eg, lower pain thresholds, attenuated pain inhibition), which is a clinical form of substantial sleep fragmentation. ${ }^{5}$ Lastly, it is also emphasized that sleep disturbances are predictive for the development of chronic pain. ${ }^{6,7}$

Summarizing these findings, the association between sleep and pain seems to become certainly obvious when sleep is profoundly altered (eg, sleep deprivation,
Correspondence: Cindy Stroemel-Scheder Department of Physiological Psychology, University of Bamberg, Markusplatz 3,

Bamberg 96045 , Germany

Tel +4995I 8632056

Email cindy.stroemel@uni-bamberg.de 
insomnia). A recent study by Karmann and colleagues aimed at enlightening the interrelation of sleep and pain in healthy subjects (without sleep or pain disorders) and found rather low associations. ${ }^{8}$ Regression analyses showed that almost no parameters of sleep had predictive value for overnight pain changes, suggesting that sleep and pain might remain uncoupled for as long both functions vary within non-pathological margins. ${ }^{9}$ Thus, the association between sleep and pain might first become apparent when there is critical variability in sleep. This begs the question how substantial alterations of sleep have to be to affect the pain system. ${ }^{9}$

One possibility to render new insights concerning the sleep and pain interrelation is the investigation of chronic pain patients and healthy controls in one sample, as such a sample shows high variability in sleep and pain parameters. The enhanced variability stems from combining healthy subjects showing sleep and pain parameters within normal range and chronic pain patients showing worse sleep and more pain than healthy controls. More precisely, patients with chronic musculoskeletal pain exhibit changes in parameters of experimental pain, eg lower pain thresholds, ${ }^{10}$ deficient conditioned pain modulation (CPM), ${ }^{11,12}$ and enhanced temporal summation (TSP) ${ }^{11,13-15}$ as well as enhanced pain catastrophizing. ${ }^{16}$ Furthermore, they show parameters suggestive of worse sleep than healthy subjects (eg, reduced total sleep times and sleep efficiency, poor sleep quality, less rapid-eye-movement sleep, and slow wave sleep). ${ }^{4,17-19}$ Since these are differences in level between chronic pain patients and healthy controls with similar between-subject variance in each group, merging both groups into one creates a sample with high betweensubject variance and, by this, favorable conditions for detecting covariations between sleep and pain.

A further objective of this study was to examine whether poor sleep is consistently associated with all indicators of pain. From a clinical perspective, sleep disturbances in chronic pain patients were found to be linked to a higher pain vulnerability and enhanced clinical pain; or in other words, poor sleepers among chronic pain patients report significantly greater clinical pain intensities than good sleepers. ${ }^{20}$ However, it remains unclear whether similar results can also be found for experimental pain parameters and pain catastrophizing.

Altogether, the aims of the present study were threefold. (1) As an advancement of the study by Karmann and colleagues, ${ }^{8}$ the main aim was to check whether sleep parameters are predictive for overnight pain changes in one sample of chronic pain patients and healthy controls. It was assumed that this heterogeneous sample exhibits sufficient inter-individual variability in sleep and pain to create favorable conditions for verifying the association of sleep and pain. (2) Additionally, it was aimed at identifying good and poor sleepers amongst chronic pain patients to check whether poor sleepers show higher levels of clinical pain, experimental pain and pain catastrophizing. (3) Lastly, a further aim was to replicate previous findings about group differences in parameters of sleep and pain between pain patients and healthy controls, with the advantage of studying these differences simultaneously in the same sample. To achieve the three research aims, pain psychophysics (pressure pain thresholds (PPT), TSP, CPM) and pain catastrophizing prior and subsequently to nights of nocturnal sleep spent at home and monitored by a portable polysomnograph were assessed in a sample of patients with chronic musculoskeletal pain and healthy controls, using the design previously described by Karmann and colleagues. ${ }^{8}$

\section{Materials and Methods}

\section{Subjects}

Twenty healthy subjects (14 female) with a mean age of 47.45 years $(S D=8.85$; range $=32-59$ years $)$ as well as 20 patients with chronic musculoskeletal pain (14 female) with a mean age of 47.25 years $(S D=9.02$; range $=25-62$ years $)$ participated in this study. Subjects were matched according to age and gender. Examined women were either postmenopausal $\left(\mathrm{n}_{\text {controls }}=5 ; \mathrm{n}_{\text {patients }}=3\right)$, using oral contraceptives $\left(\mathrm{n}_{\text {controls }}=2 ; \mathrm{n}_{\text {patients }}=3\right)$ or not using contraceptives $\left(\mathrm{n}_{\text {controls }}=7 ; \mathrm{n}_{\text {patients }}=6\right)$.

Chronic pain patients were recruited from a specialized outpatient unit for pain patients (Sozialstiftung Bamberg, Bamberg, Germany) prior to a four-week pain management program. They were required to have a diagnosis of primary musculoskeletal pain (eg, neck pain, upper back pain, low back pain or fibromyalgia; diagnoses can be found in Table 1). Tension-type headache was allowed as a secondary diagnosis. Patients were excluded if they had another predominant pain disorder, surgical interventions during the last year or if they suffered from mental or severe affective disorders. Patients were not asked to pause the intake of analgesic medication to avoid possible interferences with their pain therapy. A detailed description of medication use can be found in Table 2 .

Healthy controls were recruited via local newspaper calls and were required not to have physical or mental disorders, 
Table I Predominant Pain Sites in Patients with Chronic Musculoskeletal Pain $(n=20)$

\begin{tabular}{|l|l|l|}
\hline Diagnosis & Total & Percent \\
\hline Chronic back pain, thereof & $\mathbf{I 7}$ & $\mathbf{8 5 \%}$ \\
Neck pain & 2 & \\
Low back pain & 8 & \\
Upper back pain and low back pain & 1 & \\
Neck pain and upper back pain & 3 & \\
Neck pain and low back pain & 2 & \\
Neck pain, upper and low back pain & $\mathrm{I}$ & \\
\hline Fibromyalgia, thereof & $\mathbf{3}$ & $\mathbf{1 5 \%}$ \\
Fibromyalgia and TTH & $\mathrm{I}$ & \\
Fibromyalgia, neck pain and low back pain & $\mathrm{I}$ & \\
Fibromyalgia, TTH, neck and upper back pain & $\mathrm{I}$ & \\
\hline
\end{tabular}

Notes: Diagnoses made by MD trained in anesthesiology and pain therapy. Bold values indicate the total numbers and percental amounts of participants included in the respective diagnosis group.

Abbreviation: TTH, tension type headache.

acute or chronic pain and not to regularly use pain medication or other medications. They were instructed not to take any pain or other medications prior to and during the study period.

Ahead of the experiment, a telephone-based interview was conducted with each participant to assess possible exclusion criteria. All participants had to refrain from drinking alcohol during all testing days and gave written informed consent. Healthy subjects received $80 €$ expense allowance and $100 €$ subject reimbursement for participation; pain patients also received $80 €$ expense allowance as well as sleep and pain diagnostics as equivalent value. Psychology students could earn 15 course credits (being equivalent to $15 \mathrm{hrs}$ of study participation). The experimental protocol was approved by the ethics committee of the University of Bamberg, Germany. The study was conducted in accordance with the Declaration of Helsinki.

\section{Procedures}

Results reported here are part of a larger study dealing with the relationship between sleep and pain conducted at Bamberg University. Firstly, an overview about the entire study will be given, and then tests and measures used for the present report will be highlighted.

On the side of sleep, the study was designed to assess parameters of nocturnal sleep during two non-consecutive nights using portable polysomnography (PSG). Examined nights were one to 13 nights apart; varying intervals became necessary, as participants were required to reconcile their working schedule with study participation. During both nights, subjects were allowed to sleep at home in a familiar sleeping environment. Each night was preceded (6 p.m.) and followed

Table 2 Consumption of Analgesics and Antidepressants in Patients with Chronic Musculoskeletal Pain $(n=20)$

\begin{tabular}{|l|l|l|}
\hline Medication & Total & Percent \\
\hline None & $\mathbf{2}$ & $\mathbf{1 0 \%}$ \\
\hline Analgesics & $\mathbf{1 7}$ & $\mathbf{8 5 \%}$ \\
On demand, thereof & $\mathbf{5}$ & $\mathbf{3}$ \\
$\quad$ Nonsteroidal anti-inflammatories & 2 & $\mathbf{2 5}$ \\
$\quad$ Nonopioid analgesics & $\mathbf{4}$ & $\mathrm{I}$ \\
On demand in combination with antidepressants, thereof & $\mathrm{I}$ & $\mathbf{2 0 \%}$ \\
$\quad$ Nonsteroidal anti-inflammatories & 2 & $\mathbf{7}$ \\
$\quad$ Nonsteroidal anti-inflammatories and atypical antipsychotic & $\mathrm{I}$ \\
$\quad$ Opioid analgesics & $\mathrm{I}$ \\
As prescribed, thereof & $\mathrm{I}$ \\
$\quad$ Nonsteroidal anti-inflammatories & $\mathrm{I}$ \\
$\quad$ Nonsteroidal anti-inflammatories and nonopioid analgesics & $\mathrm{I}$ \\
$\quad$ Nonsteroidal anti-inflammatories and muscle relaxant & $\mathrm{I}$ \\
$\quad$ Nonsteroidal anti-inflammatories, anticonvulsive agent, opioid analgesics and muscle relaxant & $\mathrm{I}$
\end{tabular}

Notes: Analgesics consumed as prescribed were taken at least once a day. Bold values indicate the total numbers and percental amounts of participants taking the respective medication as given in the first column of the table. 
(8 a.m.) by laboratory sessions, which were procedurally equal and designed to assess three domains, namely cortisol, attention, and pain. At the beginning and end of each laboratory session, participants provided a saliva sample for later determination of cortisol levels. Further, two attention-related tests (dot-probe task and eye-tracking paradigm with emotional facial stimuli), which allowed to check for influences of sleep on attentional measures, and a laboratory pain testing to examine effects of sleep on pain were implemented. Pain testing consisted of the assessment of pressure pain thresholds (PPTs), followed by the assessment of temporal summation of pain (TSP) and conditioned pain modulation (CPM). After the experimental pain test, participants completed the Situational Catastrophizing Questionnaire (SCQ; state version of pain catastrophizing) $^{21}$ to assess catastrophizing thoughts in relation to the just experienced pain. At the end of the evening sessions, a portable PSG device was installed and a questionnaire to assess subjective sleep parameters (evening and morning protocols) was handed out. ${ }^{22}$ At the beginning of the morning sessions, the PSG device was detached and a further laboratory session followed. Additional questionnaires about sleep (Pittsburgh Sleep Quality Index (PSQI)) ${ }^{23}$ and pain (Pain Catastrophizing Scale (PCS); trait version of pain catastrophizing) ${ }^{24}$ as well as clinical pain (German Pain Questionnaire (DSF); assessment of pain intensity and pain duration in the sample of chronic pain patients) ${ }^{25}$ were completed at the end of the first morning session.

Since the crucial aspect of the present report is the sleep and pain interrelation, only sleep (portable polysomnography, questionnaires) and pain data (laboratory pain tests, clinical pain via self-report scales) will be reported. A detailed description of the used apparatus and methods can be found in the following sections.

\section{Assessment of Pain Parameters Questionnaires}

Situational Catastrophizing Questionnaire (SCQ)

The $\mathrm{SCQ}^{21}$ is derived from the Pain Catastrophizing Scale (PCS). ${ }^{24}$ A German version, created by our workgroup using a forward-backward-translation procedure and successfully used in previous studies, $8,26,27$ was administered immediately after each laboratory pain assessment to measure subjects' catastrophizing related to the noxious stimuli just presented. It consists of six items, eg, "I worried about when it would end", that are answered on a 5-point rating scale ranging from " 0 - not at all" to " 4 - all the time".

\section{Pain Catastrophizing Questionnaire (PCS)}

The PCS $^{24,28}$ assesses catastrophizing related to pain and consists of 13 items that can be divided into three subscales (rumination, magnification, helplessness). Items, eg, "I worry all the time about whether the pain will end", are rated on a 5-point rating scale ranging from " 0 - not at all" to " 4 - all the time". The PCS was completed at the end of the first morning session.

\section{German Pain Questionnaire (Deutscher Schmerzfragebogen, DSF)}

The $\mathrm{DSF}^{25}$ is a frequently used instrument to assess painrelated information in German-speaking pain patients. The first twelve items of the DSF were handed out to the participating pain patients at the end of the first morning session. Items ask for a description of pain with a drawing of painful body sites and assess information about duration, intensity, time course and frequency of pain, pain attacks, pain-related disability and the impact of pain on everyday life. Information about pain duration and intensity was considered for the present report. The item assessing pain duration asked for how long the patient already experiences pain. It was answered by choosing one of six given categories (duration of less than one month, one to six months, six months to a year, one to two years, two to five years, longer than five years). To assess subjective measures of pain intensity three items which ask for current pain intensity, average pain intensity and worst pain intensity during the last four weeks are answered on an 11-point rating scale ranging from " 0 - no pain" to " 10 - worst pain imaginable".

\section{Apparatus}

During the assessment of pain parameters, subjects sat in a comfortable chair. A computer-controlled pressure algometer (Noxitest Biomedical, Aalborg, Denmark; for a further description, see Nie et al) ${ }^{29}$ was used for the application of pressure stimuli (assessment of PPT and TSP, application of test stimuli (TS) in CPM-paradigm). It was placed on a table in front of the subjects. The algometer included a piston, on which a rounded aluminum footplate with a padded probe area $\left(1 \mathrm{~cm}^{2}\right)$ was fixed at the tip. The piston was moved by an electric motor. Pressure stimulation was controlled by feedback of a built-in force transducer. Pressure stimuli were applied to the middle of the fingertip of the index and middle finger of the subjects' left hand. Furthermore, a circulating water bath (WiseCircu WCB-11, Witeg $\mathrm{GmbH}$, Wertheim, Germany) was used to apply heat 
stimuli (conditioning stimulus (CS) in CPM-paradigm). The water bath contained hot water at a temperature of $46^{\circ} \mathrm{C}$, held constant by a thermostat. This temperature was selected in accordance with previous research. ${ }^{30}$ For stimulation, subjects were instructed to immerse their right hand up to the wrist into the water bath.

\section{Pressure Pain Thresholds (PPT)}

At the beginning of each laboratory session, PPTs (left index and middle finger) were assessed using the method of limits. The algometers' piston continuously applied pressure to the middle of the fingertip with a steadily increasing rate of $50 \mathrm{kPa} / \mathrm{s}$ (starting at $0 \mathrm{kPa}$ ). Subjects were given a control button and were instructed to stop the pressure increase when they first felt pain. Each time the control button was used the piston moved up and took away the pressure. After two practice trials, the described procedure was repeated for five times. The inter-stimulusinterval was $8 \mathrm{~s}$. The average of these five trials was used as an estimate of the individual PPT. For later analyses, PPTs of index and middle finger were averaged.

\section{Temporal Summation of Pain (TSP)}

The further laboratory session consisted of two blocks (baseline: no CS applied; CPM: CS applied). During both blocks, TSP was assessed. TSP was tested by comparing sensations evoked by single pulses of pressure to sensations evoked by series of five pulses (only the last pulse of pressure was rated). The series of five pulses was applied with a repetition frequency of $0.5 \mathrm{~Hz}$. Overall three single pulses and three series of five pulses were presented in an alternating fashion with an inter-stimulus-interval of $60 \mathrm{~s}$. The six stimulation episodes ( 3 single pulses, 3 series) were presented once in each of the two experimental blocks (baseline, CPM). During the first block (baseline), pressure stimuli were applied to the left index finger, during the second block (CPM) to the left middle finger. Stimulus intensity was adjusted to the subjects' PPT $(50 \%$ above threshold of respective finger) and increased with a rate of rise of $75 \%$ of the target intensity per second. A safety limit was set at 750 $\mathrm{kPa}$, which allowed no stimulation above this level. The stimuli had a saw-tooth shape with stimulus duration at maximum intensity of $0.1 \mathrm{~s}$.

Subjects were instructed to verbally report perceived pain intensity using a numerical rating scale ranging from "0 - no pain" to " 10 - extremely strong pain".

TSP scores were calculated as follows: The three numerical ratings of the sensation evoked by a train of pulses and the three numerical ratings of the sensation evoked by single pulses were separately averaged. Then, the difference between the average rating of the train of pulses and the average rating of single pulses was calculated. Thus, higher scores indicate stronger TSP. TSP scores used for further evaluation were computed using data of baseline-condition only.

\section{Conditioned Pain Modulation (CPM)}

During the second experimental block, the CPM-effect was tested using water of painful heat $\left(46^{\circ} \mathrm{C}\right)$ as conditioning stimulus (CS) and pressure stimuli as test stimuli (TS; same pressure stimuli as used for TSP assessment). Perceived pain intensity of TS was supposed to be modulated by the CS. Subjects were instructed to now rate both the pressure pulses and the heat stimuli (ratings immediately given after application of the pressure pulse(s)), using the same rating scale as described before. To assess the CPM-effect, ratings of pressure stimuli (TS) while the subjects' right hand was immersed into hot water (CS) were compared to the ratings during baseline, when no CS was applied.

CPM-effects were calculated as follows: Differences were calculated between the average pain rating for TS and the average pain rating for TS while the CS was applied, each separately for ratings of single pulses and ratings of series of pulses. Both differences were in turn averaged, resulting in a single value for CPM, with positive values indicating pain inhibition and negative values indicating pain facilitation.

\section{Assessment of Sleep Parameters Apparatus}

PSG recordings were conducted using the SOMNOwatch ${ }^{\mathrm{TM}}$ plus EEG6 (SOMNOmedics, Randersacker, Germany) and prepared in the laboratory of the University at the end of the evening sessions. Four EEG-channels (C3, C4, O1, O2), bilateral electrooculogram (EOG; left and right) and two channels of electromyogram (EMG; M. submentalis) were recorded using gold disc electrodes (Grass Technologies, West Warwick, USA). Positioning of EEG-electrodes was based on the international 10-20-system. All electrodes were referenced towards $\mathrm{Cz}$. Before attaching electrodes, skin was cleaned with cleansing gel (Nuprep, Weaver and Company, Aurora, USA) to reduce electrode resistance. Electrodes were attached using electrode cream (EC2 Electrode Cream, Grass Technologies, West Warwick, USA) and fixed using a piece of mull and a plaster. After installing the 
PSG device, subjects left the laboratory and slept at home.

They were instructed to follow their regular sleeping habits.

PSG recordings were analyzed according to the standard PSG protocol. ${ }^{31}$ First, the DOMINO light software (SOMNOmedics, Randersacker, Germany) automatically scored sleep and wake stages in epochs of 30s, then a visual inspection was performed to check whether the automatic analysis performed correctly according to the Rechtschaffen and Kales criteria. In case of discrepancy, the automatic analysis was overruled and stages were rescored. The following sleep parameters were further examined: Total sleep time (TST; defined as the time between "lights off" and "lights on" without sleep onset latency and time spent awake), sleep efficiency (SE; total sleep time/time spent in bed after sleep onset * 100\%), sleep onset latency (SL; time from "lights off" to the first appearance of non-REM stage 2), total number and total duration of awakenings as well as durations of rapid eye movement sleep (REM sleep), non-REM stages 1 and 2, and slow wave sleep (SWS; non-REM stages 3 and 4).

\section{Questionnaires}

\section{Evening and Morning Protocols}

Evening and morning protocols ${ }^{22}$ were used to assess selfreported sleep quality. Only the morning protocol was used for further evaluation. It was completed after waking up in the morning and covers the night and wellbeing in the morning. Overall four items were used: To assess the subjective state in the morning three items dealing with mood (ranging from "depressed" to "untroubled"), freshness (ranging from "run down" to "refreshed") and tension (ranging from "tense" to "relaxed") were answered on a 6-point scale, and a question about restfulness of sleep was answered on a 5-point scale (ranging from "very restful" to "not restful at all").

\section{Pittsburgh Sleep Quality Index (PSQI)}

The PSQI ${ }^{23}$ deals with several aspects of regular sleeping habits during the past four weeks. It asks for the time subjects usually go to bed and get up, sleep onset latency and sleep duration. It as well covers problems with sleep onset, frequent awakenings, and quality of sleep. It was completed at the end of the first morning session. Seven indices (sleep quality, sleep latency, sleep duration, sleep efficiency, sleep disorders, sleep medication use, daytime sleepiness) and one overall index (scores $\leq 5$ indicating good sleep) can be derived. All indices were used for further evaluation.

\section{Statistical Analysis}

Data were analyzed using SPSS version 22 (IBM Corporation, Armonk, NY, USA). Significance level was set at $\alpha=5 \%$. Data are presented as mean and standard deviation. Bonferroni corrections for multiple testing were applied.

\section{Prediction of Overnight Pain Changes by Sleep Parameters}

Hierarchical regression analyses were calculated to predict overnight changes in experimental pain by sleep parameters. Solely experimental pain parameters were considered as these could be assessed in both pain patients and healthy subjects, allowing for sufficient statistical power. Scores for pain changes $\left(\mathrm{PPT}_{\text {Diff }}, \mathrm{TSP}_{\text {Diff, }}, \mathrm{CPM}_{\text {Diff, }}, \mathrm{SCQ}_{\text {Diff }}\right)$ were calculated as the difference between morning and evening scores. Negative values represent higher pain-scores in evening versus morning sessions (overnight decrease); positive values accordingly indicate an increase. Variables "night" (night 1, night 2) and "group" (pain patients, healthy controls) were entered as dummy-coded variables. Sleep parameters (predictors) were grouped into three domains according to their functional and content-related similarity: (1) general PSG (TST, SE, SL, number and duration of awakenings), (2) sleep stage-specific PSG (durations of nonREM stages 1 and 2, REM sleep, SWS) and (3) subjective sleep quality ("mood", "freshness", "tension" and "restfulness" items). Accordingly, for all four difference-scores, three regression analyses were calculated, with "night", "group" and additionally either domain (1), (2), or (3) as predictors, resulting in overall 12 regression analyses. The approach to conduct several regression analyses with keeping different domains of predictors separately was chosen in face of the relatively small sample size and performed in accordance with the previous study. ${ }^{8}$ Thus, not too many predictors were included in one regression analysis.

\section{Comparison of Good and Poor Sleepers}

It was further checked whether poor sleepers among the chronic pain patients were more pain vulnerable than good sleepers. Patients were grouped relying on objective sleep quality measures (mean sleep efficiency of both measured nights), using the cut-off of $\geq 85 \%$ indicating good sleep (Morin, 1993, as cited by O'Donoghue et al). ${ }^{32}$ Analyses of variance (ANOVAs) were conducted with betweensubject factor "group" (good sleepers, poor sleepers). For clinical pain ("pain intensity level") within-subject factor "DSF-item" (current pain intensity, average, and worst 
pain intensity during the past four weeks; as measured by the DSF) was used. For experimental pain parameters (PPT, TSP, CPM) and situational pain catastrophizing (SCQ) within-subject factor "session" (evening 1, morning 1 , evening 2, morning 2) was considered, with separate ANOVAs for each pain parameter.

\section{Comparisons of Sleep and Pain Parameters between Chronic Pain Patients and Healthy Controls}

A multivariate ANOVA for sleep parameters with withinsubject factor "night" (night 1 , night 2 ) and between-subject factor "group" (pain patients, healthy controls) was calculated to check for differences in sleep parameters (TST, SE, SL, total number and duration of awakenings, durations of non-REM stages 1 and 2, SWS, REM-sleep, subjective parameters). To check for group differences in pain parameters (PPT, TSP, CPM, SCQ) four separate repeated measurements ANOVAs with within-subject factors "night" (night 1, night 2) and "daytime" (evening, morning) and between-subject factor "group" (pain patients, healthy controls) were calculated. Separate ANOVAs were conducted instead of one MANOVA to not include too many parameters in one analysis. Due to directed hypotheses according to between-group comparisons, onetailed significance is reported in this case.

Power analyses were calculated using G-Power 3.1. ${ }^{33}$ For multivariate ANOVAs (sleep parameters), sample sizes of $n=20$ to $n=25$ per group were needed to reach a significance level of $\alpha=0.05$ with a power of $80 \%$ for medium effect sizes. For repeated-measures ANOVAs (pain parameters) including two groups and four measurements, sample sizes of $n=17$ to $n=29$ per group were needed. Hence, a sample of $n=20$ per group was assessed.

\section{Results}

Pain patients and healthy controls did not differ according to age (healthy controls: $M=47.45$ years, $S D=8.85$; patients: $M=47.25$ years, $S D=9.02 ; t_{38}=0.071, p=$ $0.944, d=-0.022$ ) and gender (14 females in both groups). Descriptive data derived from pain and sleep questionnaires (DSF, PCS, PSQI) can be found in Table 3. Scores of PCS and PQSI, as well as their subscales, were within normal range in healthy subjects and beyond normal in pain patients. ${ }^{23,24}$ Summarizing main results of the DSF, patients' current pain intensity was $M=5.43(S D=2.47)$ and pain duration were once half a year, three times between half a year and one year, four times between one and two years, five times between two and five years, and seven times longer than five years.
Table 3 Descriptive Data of Assessed Sleep and Pain Questionnaires in Patients with Chronic Pain and Pain-Free Participants

\begin{tabular}{|c|c|c|c|}
\hline & $\begin{array}{l}\text { Both } \\
\text { Groups }\end{array}$ & Patients & Controls \\
\hline \multicolumn{4}{|l|}{ DSF (Pain) } \\
\hline Current Pain Intensity & - & $5.43(2.47)$ & - \\
\hline Average Pain Intensity & - & $5.95(2.33)$ & - \\
\hline Worst Pain Intensity & - & $7.85(1.98)$ & - \\
\hline \multicolumn{4}{|c|}{ PCS (Habitual Pain Catastrophizing) } \\
\hline Pain Catastrophizing & $20.95(9.7 I)$ & $25.38(10.16)$ & $17.10(7.59)$ \\
\hline Rumination & $7.55(3.48)$ & $8.40(3.91)$ & $6.70(2.83)$ \\
\hline Magnification & $4.70(2.69)$ & $5.45(3.19)$ & $3.95(1.88)$ \\
\hline Helplessness & $8.93(4.69)$ & II.53 (4.47) & $6.45(3.61)$ \\
\hline \multicolumn{4}{|c|}{ PSQI (Habitual Sleep) } \\
\hline Sleep Quality & $1.35(0.89)$ & $1.95(0.76)$ & $0.75(0.55)$ \\
\hline Sleep Latency & $1.28(1.04)$ & $1.70(1.08)$ & $0.85(0.8 I)$ \\
\hline Sleep Duration & $0.80(0.99)$ & $1.20(1.11)$ & $0.40(0.68)$ \\
\hline Sleep Efficiency & $0.90(1.15)$ & $1.35(1.27)$ & $0.45(0.38)$ \\
\hline Sleep Disorders & $1.23(0.58)$ & $1.55(0.61)$ & $0.90(0.31)$ \\
\hline Sleep Medication Use & $0.30(0.82)$ & $0.60(1.10)$ & $0.00(0.00)$ \\
\hline Daytime Sleepiness & $1.40(0.84)$ & $1.90(0.64)$ & $0.90(0.72)$ \\
\hline PSQI-Score & $7.25(4.36)$ & $10.25(3.60)$ & $4.25(2.67)$ \\
\hline
\end{tabular}

Notes: Pain Intensity: $\min =0, \max =10$; Average and Worst Pain Intensity during the last four weeks. Pain Catastrophizing: $\min =0, \max =52$; Rumination: $\min =0$, $\max =16$; Magnification: $\min =0, \max =12$; Helplessness: $\min =0, \max =24$; All subscales ranging from $\min =0$ to $\max =3$. PSQI-Score: $\min =0, \max =21$. Results are presented as $M(S D)$.

Abbreviations: DSF, Deutscher Schmerzfragebogen (German Pain Questionnaire); PCS, Pain Catastrophizing Questionnaire; PSQI, Pittsburgh Sleep Quality Index.

\section{Prediction of Overnight Pain Changes by Sleep Parameters}

Hierarchical regression analyses were calculated to predict overnight pain changes by sleep parameters; overnight pain changes can be taken from Table 4. Of overall 12 regression analyses, only two reached statistical significance. $\mathrm{PPT}_{\text {Diff }}$ was predicted by "night" $\left(R^{2}=0.060, F=5.012, p=0.028\right)$ and $\mathrm{SCQ}_{\text {Diff }}$ by subjective parameters of sleep $\left(R^{2}=0.179\right.$, $F=2.644, p=0.022$ ), but both results were insignificant after Bonferroni correction (Bonferroni corrected $\alpha$ 's: $\alpha=0.025$ and $\alpha=0.008$, respectively). Results therefore indicate no prediction of overnight pain changes by the different domains of sleep predictors.

\section{Comparison of Good and Poor Sleepers}

Descriptive data of parameters of clinical pain, experimental pain as well as pain catastrophizing of good and poor sleepers can be taken from Table 5. Despite poor sleepers descriptively showing higher pain intensities, lower pain 
Table 4 Differences (Morning Minus Evening) of Pain Parameters

\begin{tabular}{|c|c|c|c|}
\hline & Difference I & Difference 2 & Both \\
\hline \multicolumn{4}{|l|}{$\mathrm{PPT}_{\text {Diff }}$} \\
\hline Patients & $-21.69(80.16)$ & $-2.53(29.75)$ & $-12.11(60.47)$ \\
\hline Controls & $-32.23(34.30)$ & $-2.23(36.77)$ & $-17.23(38.24)$ \\
\hline Both Groups & $-26.96(61.09)$ & $-2.38(33.02)$ & $-14.67(50.33)$ \\
\hline \multicolumn{4}{|l|}{$\mathrm{TSP}_{\text {Diff }}$} \\
\hline Patients & $0.28(1.35)$ & $0.13(0.7 I)$ & $0.21(1.07)$ \\
\hline Controls & $0.33(0.77)$ & $-0.22(0.83)$ & $0.06(0.83)$ \\
\hline Both Groups & $0.31(1.08)$ & $-0.04(0.78)$ & $0.13(0.96)$ \\
\hline \multicolumn{4}{|l|}{$C P M_{\text {Diff }}$} \\
\hline Patients & $0.12(1.72)$ & $-0.10(0.81)$ & $0.01(1.33)$ \\
\hline Controls & $-0.29(1.42)$ & $-0.23(1.03)$ & $-0.23(1.23)$ \\
\hline Both Groups & $-0.09(1.57)$ & $-0.17(0.92)$ & $-0.11(1.28)$ \\
\hline \multicolumn{4}{|l|}{$S C Q_{\text {Diff }}$} \\
\hline Patients & $-1.15(4.08)$ & $-0.03(1.81)$ & $-0.59(3.17)$ \\
\hline Controls & $-0.60(2.33)$ & $-0.25(1.59)$ & $-0.43(1.97)$ \\
\hline Both Groups & $-0.88(3.29)$ & $-0.14(1.69)$ & $-0.51(2.62)$ \\
\hline
\end{tabular}

Notes: Difference I refers to overnight pain changes (morning minus evening) during the first assessed night, difference 2 accordingly refers to overnight pain changes during the second night. Results are presented as $M(S D)$.

Abbreviations: PPT, Pressure Pain Threshold; TSP, Temporal Summation of Pain; CPM, Conditioned Pain Modulation; SCQ, Situational Pain Catastrophizing.

Table 5 Descriptive Data of Clinical Pain Intensities (as Measured by the DSF), Experimental Pain Parameters and Situational Pain Catastrophizing in Good and Poor Sleepers

\begin{tabular}{|l|l|l|}
\hline \multirow{2}{*}{} & \multicolumn{2}{|l|}{ PSG } \\
\cline { 2 - 3 } & Poor Sleepers & Good Sleepers \\
\hline Clinical Pain & & \\
Current Pain Intensity & $6.35(2.40)$ & $4.50(2.27)$ \\
Average Pain Intensity & $6.30(2.11)$ & $5.60(2.59)$ \\
Worst Pain Intensity & $8.00(2.21)$ & $7.70(1.83)$ \\
\hline Experimental Pain & & \\
PPT & $172.69(112.14)$ & $184.84(54.28)$ \\
TSP & $1.09(0.53)$ & $0.73(0.59)$ \\
CPM & $-0.12(0.8 I)$ & $-0.27(0.48)$ \\
\hline Situational Pain & & \\
Catastrophizing & & $4.95(3.60)$ \\
SCQ & $6.63(3.78)$ & \\
\hline
\end{tabular}

Notes: Good $(n=10)$ and poor sleepers $(n=10)$ as classified by polysomnographically assessed sleep efficiency; Pain Intensity: $\min =0, \max =10$; Average and Worst Pain Intensity during the last four weeks. Results are presented as $M(S D)$.

Abbreviation: PSG, Polysomnography; PPT, Pressure Pain Threshold; TSP, Temporal Summation of Pain; CPM, Conditioned Pain Modulation; SCQ, Situational Pain Catastrophizing; DSF, Deutscher Schmerzfragebogen (German Pain Questionnaire).

thresholds and enhanced catastrophizing than good sleepers, none of the conducted variance analyses reached significance (all $p$ 's $>0.09$ ). Thus, results did not prove differences between good and poor sleepers (as categorized by polysomnographically assessed sleep efficiency) according to measures of clinical pain, experimental pain and situational pain catastrophizing.

\section{Comparisons of Sleep and Pain Parameters Between Chronic Pain Patients and Healthy Controls Sleep}

Descriptive data of sleep parameters can be found in Supplementary Tables 1-3. There were overall 13 missing values in the morning protocol (all due to non-compliance with instructions), which were imputed using mean substitution. As expected, ANOVA yielded no significant main effect of "night" $\left(F(13,64)=0.381, p=0.971, \eta^{2}=0.072\right)$, indicating no differences between both nights. There was a significant effect of factor "group" $(F(13,64)=2.513, p=0.008$, $\eta^{2}=0.338$ ). Post hoc $t$-tests revealed that healthy controls and patients differed significantly according to their TST $\left(t_{78}=2.370, p=0.010, d=-0.530\right), \mathrm{SE}\left(t_{78}=2.176 p=0.017\right.$, $d=-0.486)$ and the time awake during the night $\left(t_{78}=-2.319\right.$, $p=0.012, d=0.518$ ). Pain patients slept shorter, showed lower sleep efficiency and spent more time awake during the night. Furthermore, pain patients reported significantly worse sleep according to all subjective sleep parameters, namely impaired mood in the morning ( $\left.t_{78}=4.139, p<0.001, d=-0.93\right)$, less freshness $\left(t_{78}=3.189, p=0.002, d=-0.62\right)$, enhanced tension $\left(t_{78}=4.719, p<0.001, d=-1.065\right)$ and lower restfulness of sleep $\left(t_{78}=3.359, p=0.001, d=-0.748\right)$.

\section{Pain}

Descriptive data of assessed pain parameters (PPT, TSP, CPM, SCQ) can be found in Figure 1. CPM was assessed in 19 of 20 patients because one did not tolerate the water bath of $46^{\circ} \mathrm{C}$ temperature. There were overall three missing SCQ-values because the questionnaire was not fully completed, which were imputed using mean substitution.

ANOVA for PPT revealed a significant effect of factor "group" $\left(F(1,37)=3.178, p=0.042, \eta^{2}=0.077\right)$. Pain patients had significantly lower PPTs both in the evening $\left(t_{78}=2.386, p=0.010, d=-0.649\right)$ and morning testing sessions $\left(t_{78}=2.502, p=0.007, d=-0.559\right)$ than healthy controls. Additionally, a significant effect of factor "daytime" $\left(F(1,38)=5.737, p=0.022, \eta^{2}=0.131\right)$ and a significant interaction of "daytime $\mathrm{x}$ night" $(F(1,38)=$ 6.292, $p=0.017, \eta^{2}=0.142$ ) were found. PPTs were significantly higher in the evening (versus morning) of the first testing session $\left(t_{39}=2.791, p=0.008, d=-0.216\right)$. 

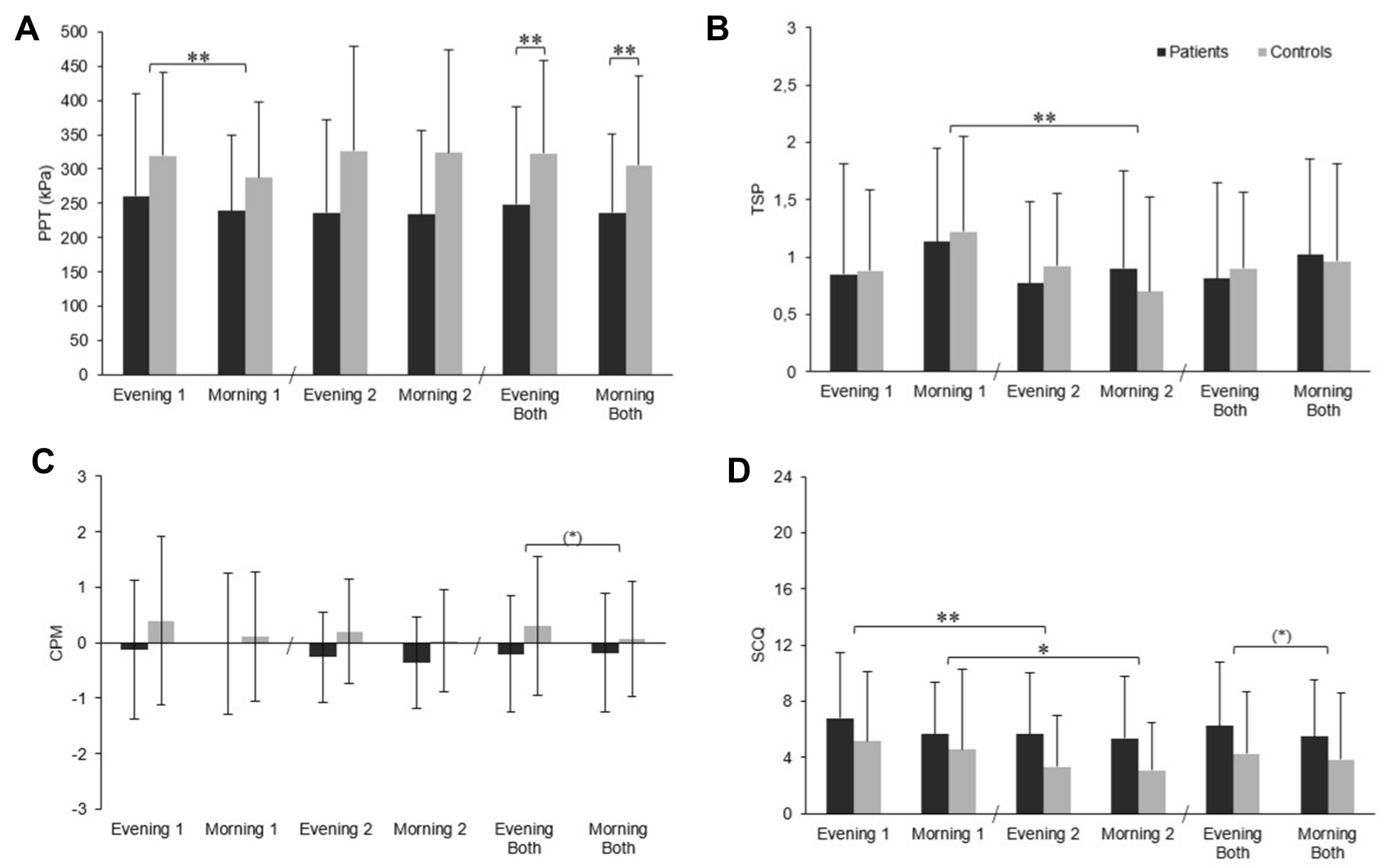

Figure I Parameters of pain depicted for all testing sessions separately and comprised for both evening and morning testing sessions.

Notes: (A) Pressure Pain Threshold (PPT). (B) Temporal Summation (TSP). (C) Conditioned Pain Modulation (CPM). (D) Situational Pain Catastrophizing (SCQ). ${ }^{*}$ P $<<0$. ; $*_{p} \leq 0.05 ; * * p \leq 0.01$.

This difference vanished for the latter assessments before and after the second night.

ANOVA for TSP revealed a significant effect of factor "night" $\left(F(1,38)=5.446, p=0.025, \eta^{2}=0.125\right)$. TSP was significantly higher in the first than in the second morning $\left(t_{39}=3.237, p=0.002, d=-0.463\right)$. No other effects were found to be significant (all $p$ 's $>0.1$ ). It was additionally checked whether TSP generally resulted in an increase of pain perception. For that purpose, deviations of the differences between ratings of single pulses and ratings of series of pulses from 0 (0 indicating no increase in pain perception due to repeated stimuli) were tested by using one-sample $t$-tests and found to be significant in both groups, suggesting an increase in pain perception due to TSP (healthy subjects: $t_{39}=9.225$, $p<0.001, d=-2.063$; patients; $t_{39}=9.026, p<0.001$, $d=-2.018$ ).

ANOVA for CPM revealed a near-significant effect of "group" $\left(F(1,37)=2.137, p=0.076, \eta^{2}=0.055\right)$, indicating at best a trend to reduced CPM in the pain patients. All other effects did also not reach significance $(\mathrm{p}>0.09)$.
ANOVA for SCQ revealed a near-significant effect of "group" $\left(F(1,38)=2.004, p=0.083, \eta^{2}=0.050\right)$, indicating at best a trend to more situational pain catastrophizing in pain patients. Additionally, a near-significant effect of the factor "night" was found $\left(F(1,38)=2.614, p=0.057, \eta^{2}=0.136\right)$. SCQ was higher in the first evening session than in the second evening session $\left(t_{39}=2.775, p=0.004, d=-0.363\right)$. This difference was also present when comparing morning testing sessions $\left(t_{39}=1.691, p=0.050, d=-0.199\right)$. This pattern suggests a de-catastrophizing action of repeated sessions in a pain laboratory.

\section{Discussion}

In the present study, parameters of sleep and experimental pain in a sample of healthy subjects and patients with chronic musculoskeletal pain were examined. In the pain patients, additionally clinical pain was assessed. (1) The main aim of the study was to check whether sleep parameters are predictive for overnight pain changes. The combined sample of healthy subjects and chronic pain patients was chosen to examine this research question, 
because it offered the needed variance in sleep and pain, which allowed to check for a prediction of pain by sleep parameters. Additional aims included (2) investigating whether poor sleepers among the pain patients are more pain vulnerable than good sleepers and (3) replicating that pain patients show worse sleep and deviant experimental pain parameters than healthy subjects.

\section{Prediction of Overnight Pain Changes by Sleep Parameters}

Of overall 12 regression analyses to predict overnight pain changes by sleep parameters, the only two reaching significance (prediction of $\mathrm{PPT}_{\text {Diff }}$ by "night" and $\mathrm{SCQ}_{\text {Diff }}$ by subjective sleep quality) did not withstand Bonferroni correction. This indicates that the different domains of sleep parameters had no predictive value for overnight changes in pain processing, even in our sample with a wide variability concerning sleep and pain (from normality to mild-to-moderate pathology). It is therefore reasonable to assume that the association between sleep and pain is less easily established than demonstrated by previous studies, which mainly used drastic intra-individual sleep manipulations (eg, sleep deprivation). ${ }^{1-3}$

The main idea of the present study was to further clarify the inter-individual variability needed in sleep and pain for both systems to correlate. A previous study examining healthy, pain-free normal sleepers highlighted that sleep and pain appeared mainly uncorrelated in this sample with low between-subject variability. ${ }^{8}$ The present study used the same experimental design as the former one, but examined chronic pain patients in addition to healthy subjects, while assuming that the combined sample shows greater between-subject variability than a sample consisting solely of healthy subjects. Indeed, an enhanced variance was observed, but results of regression analyses suggest that this variance was still not sufficient for proving correlations between both systems. A possible conclusion is that an association might only become apparent in pathological or experimentally induced forms of variance (eg, hyperalgesia in sleep disorders, pain changes following experimental sleep deprivation). ${ }^{8}$ This may also indicate that the assumption of an ubiquitous covariation between sleep and pain might be incorrect and/or that betweensubject designs are inappropriate to prove this covariation. ${ }^{9}$

\section{Comparison of Good and Poor Sleepers}

An additional aim of the present study was to examine whether poor sleepers among the chronic pain patients are more pain vulnerable than good sleepers. Pain patients were classified via objective measures of sleep quality (polysomnographical sleep efficiency). Poor sleepers consistently exhibited descriptively higher clinical pain intensities; however, none of the group differences according to clinical pain reached significance. Furthermore, there were no significant differences between good and poor sleepers according to parameters of experimental pain (pressure pain thresholds, temporal summation of pain, conditioned pain modulation) and situational pain catastrophizing. Whereas previous studies solely relied on self-reported sleep quality to group pain patients into good and poor sleepers, ${ }^{20}$ the present study used objective sleep quality to differentiate the two groups. Although sleep disturbances and pain complaints are indeed co-occurring in chronic pain patients, the impact of moderately poor sleep on pain (clinical and experimental pain) seems to be less reliable as suggested by previous studies, ${ }^{20,32,34}$ which mainly highlighted associations/correlations between subjective measures of sleep quality and clinical pain. Additionally, the sample size might have been too small to detect subtle relationships between pain and sleep.

\section{Comparisons of Sleep and Pain Parameters Between Chronic Pain Patients and Healthy Controls}

A further aim was the replication of well-known group differences in sleep and pain processing parameters when comparing pain-free individuals and patients with chronic pain.

\section{Sleep}

Corresponding to previous findings, sleep in pain patients was significantly worse than sleep of healthy controls according to general PSG parameters (TST, SE, time awake) ${ }^{17,18,32,35}$ and subjective sleep quality. ${ }^{17,32,35}$ This reflects difficulties in maintaining undisturbed sleep during the time spent in bed and an impairment of perceived sleep quality in patients with chronic musculoskeletal pain. ${ }^{17,36,37}$ Contrary to other findings, ${ }^{4,19}$ there were no alterations in the durations of the different sleep stages (nREM stages 1 and 2, REM sleep, SWS) in the pain patients. However, research about sleep architecture changes in patients with chronic musculoskeletal pain is not unequivocal, ${ }^{17}$ as some earlier studies also only showed insignificant differences in the amounts of different sleep stages between healthy subjects and pain patients. ${ }^{18,38}$ In our study, the time pain patients spent in different sleep stages seemed to be maintained. In summary, in the present study 
typical, well-established changes in sleep parameters of chronic pain patients were replicated.

\section{Pain}

Pain patients exhibited significantly lower PPTs than healthy controls, which corroborates results of previous studies ${ }^{10}$ and can be seen as a robust marker of mechanical hyperalgesia in chronic musculoskeletal pain.

Contrary to previous experimental studies in patients with chronic musculoskeletal pain (ie, fibromyalgia patients), there was no significantly enhanced TSP in our chronic pain patients. ${ }^{39-43}$ As pain sensitivity in fibromyalgia is markedly enhanced when compared to patients with mild and severe chronic low back pain (as mainly investigated in our study), ${ }^{44}$ the necessity to further examine TSP in chronic musculoskeletal pain patients arises, to check whether TSP differs in patients with regional and widespread pain.

At best as a trend, our pain patients exhibited reduced CPM as compared to healthy controls, but this group difference did not reach statistical significance. This conflicts with previous results showing impaired CPM in various chronic musculoskeletal pain populations. ${ }^{11,12,45}$ The CPM-paradigm used in the present study has proven to be valid in recent studies, making a methodological explanation of the discrepant results unlikely. $8,30,46$

Lastly, our pain patients reported - also only as a trend - enhanced situational pain catastrophizing as compared to healthy controls, which is in accordance with previous findings. ${ }^{16}$ The insignificant difference might be due to bottom effects, as both groups in the present study did not seem to experience experimental pain stimuli as overly burdensome, resulting in low SCQ ratings.

\section{Strengths and Weaknesses}

To start with the characteristics of our study, which may be seen as strengths, the following considerations should be highlighted: As a statistical prerequisite of determining the predictive power of sleep parameters for experimental pain, it was aimed at gaining sufficient inter-individual variance. Accordingly, both healthy subjects with regular patterns of sleep and pain and chronic pain patients with patterns deviating from normality were assessed, thus creating high between-subject variability in one sample. As a further measure to enhance statistical power, two nights of sleep were assessed. There were no significant within-group differences over the two nights regarding sleep parameters and overnight changes in pain parameters. This allowed considering the nights as replications and strengthening statistical power by including both examined nights in data evaluation.

One shortcoming of the present study, as mentioned before, is the relatively small sample size. The study design consisting of overall four precisely timed laboratory sessions (each lasting approximately two hours) and two nights of ambulant polysomnography was associated with enormous effort for participants (eg, postponing working hours to get to the laboratory on time or managing to get several days off). This made the recruitment of subjects, especially of pain patients, difficult to accomplish. However, in light of conducted power analyses, sample sizes of twenty subjects per group approach the best possible solution.

Furthermore, the study design made the implementation of evening (6 p.m.) and morning ( 8 a.m.) pain testing sessions at fixed times necessary. Because sleep and time of day are in part confounded by nature, diurnal effects on pain perception besides the effects of the sleep-wake cycle might as well have played a role. Therefore, differences between evening and morning testing sessions might either be due to sleep in between those sessions or due to diurnal variations in pain processing. Previous findings concerning diurnal variations in pain processing revealed inconsistent results, ${ }^{47}$ making it difficult to account for it in further design planning.

Additionally, overnight changes in clinical pain were not assessed, since this measure in patients mainly suffering from musculoskeletal back pain could be expected to have too little variance to be suitable for correlation analysis. Therefore, clinical pain was considered only in the comparison of good and poor sleepers amongst the pain patients. Also, participating pain patients were not asked to pause their medication intake to minimize possible interferences with their pain treatment. Different medications, such as antidepressants or opioids, are known to influence sleep differently, ${ }^{17,18,48}$ but due to the small sample size, a separate analysis of subjects taking specific kinds of medication was not feasible. However, it must be considered that the majority of patients with chronic pain regularly use medication, which in turn underlines the external validity of the present study for examining chronic pain patients under everyday-conditions.

\section{Conclusion}

In accordance with previous research, chronic pain patients showed worse sleep parameters and lower pressure pain thresholds than healthy controls. Among the chronic pain patients, no differences in clinical pain 
measures were found between good and poor sleepers. As a main objective, this study systematically examined whether parameters of sleep are predictive for overnight pain changes in a combined sample of healthy subjects and patients with chronic musculoskeletal pain, which was supposed to show sufficient variance to qualify for covariance analyses. However, sleep parameters were hardly apt to predict overnight pain changes, leaving the association of both systems mainly unproven when using between-subject variance for verification, even when patients with chronic pain were included in the sample.

\section{Funding}

This study was financially supported by Dr. Robert-PflegerStiftung Bamberg.

\section{Disclosure}

The authors report no conflicts of interest in this work.

\section{References}

1. Lautenbacher S, Kundermann B, Krieg J-C. Sleep deprivation and pain perception. Sleep Med Rev. 2006;10(5):357-369. doi:10.1016/j. smrv.2005.08.001

2. Kundermann B, Krieg J-C, Schreiber W, Lautenbacher S. The effect of sleep deprivation on pain. Pain Res Manag. 2004;9(1):25-32. doi: $10.1155 / 2004 / 949187$

3. Karmann AJ, Kundermann B, Lautenbacher S. Schlafentzug und Schmerz: Ein Review der neuesten Literatur. Schmerz. 2014;28 (2):141-146. doi:10.1007/s00482-014-1394-6

4. Roehrs TA, Roth T. Sleep and pain: interaction of two vital functions. Semin Neurol. 2005;25(1):106-116. doi:10.1055/s-2005-867079

5. Haack M, Scott-Sutherland J, Santangelo G, Simpson NS, Sethna N, Mullington JM. Pain sensitivity and modulation in primary insomnia. Eur J Pain. 2012;16(4):522-533. doi:10.1016/j.ejpain.2011.07.007

6. Davies KA, Macfarlane GJ, Nicholl BI, et al. Restorative sleep predicts the resolution of chronic widespread pain: results from the EPIFUND study. Rheumatology (Oxford). 2008;47(12):1809-1813. doi:10.1093/rheumatology/ken389

7. Nitter AK, Pripp AH, Forseth K. Are sleep problems and non-specific health complaints risk factors for chronic pain? A prospective population-based study with 17 year follow-up. Scand J Pain. 2012;3(4):210-217. doi:10.1016/j.sjpain.2012.04.001

8. Karmann AJ, Lauer C, Ziegler E, Killian L, Horn-Hofmann C, Lautenbacher S. Associations of nocturnal sleep with experimental pain and pain catastrophizing in healthy volunteers. Biol Psychol. 2018;135:1-7. doi:10.1016/j.biopsycho.2018.02.015

9. Lautenbacher S. Sleep and pain are definitely coupled-but how tight is this coupling? Pain. 2017;159(1):3-4. doi:10.1097/j.pain.0000000000001082

10. Ge H-Y, Vangsgaard S, Omland Ø, Madeleine P, Arendt-Nielsen L. Mechanistic experimental pain assessment in computer users with and without chronic musculoskeletal pain. BMC Musculoskelet Disord. 2014;15:412. doi:10.1186/1471-2474-15-412

11. Arendt-Nielsen L, Fernández-de-Las-Peñas C, Graven-Nielsen T. Basic aspects of musculoskeletal pain: from acute to chronic pain. J Man Manip Ther. 2011;19(4):186-193. doi:10.1179/106698111X13129729551903

12. Potvin S, Marchand S. Pain facilitation and pain inhibition during conditioned pain modulation in fibromyalgia and in healthy controls. Pain. 2016;157(8):1704-1710. doi:10.1097/j.pain.0000000000000573
13. Arendt-Nielsen L, Graven-Nielsen T. Central sensitization in fibromyalgia and other musculoskeletal disorders. Curr Pain Headache Rep. 2003;7:355-361. doi:10.1007/s11916-003-0034-0

14. Graven-Nielsen T, Arendt-Nielsen L. Peripheral and central sensitization in musculoskeletal pain disorders: an experimental approach. Curr Rheumatol Rep. 2002;(4):131-321. doi:10.1007/s11926-0020040-y

15. Owens MA, Bulls HW, Trost Z, et al. An examination of pain catastrophizing and endogenous pain modulatory processes in adults with chronic low back pain. Pain Med. 2016;17(8):1452-1464. doi:10.1093/pm/pnv074

16. Campbell CM, Kronfli T, Buenaver LF, et al. Situational versus dispositional measurement of catastrophizing: associations with pain responses in multiple samples. J Pain. 2010;11(5):443-453.e2. doi:10.1016/j.jpain.2009.08.009

17. Bjurstrom MF, Irwin MR. Polysomnographic characteristics in nonmalignant chronic pain populations: a review of controlled studies. Sleep Med Rev. 2016;26:74-86. doi:10.1016/j.smrv.2015.03.004

18. Okura K, Lavigne GJ, Huynh N, Manzini C, Fillipini D, Montplaisir JY. Comparison of sleep variables between chronic widespread musculoskeletal pain, insomnia, periodic leg movements syndrome and control subjects in a clinical sleep medicine practice. Sleep Med. 2008;9(4):352-361. doi:10.1016/j.sleep.2007.07.007

19. Jennum P, Jensen R. Sleep and headache. Sleep Med Rev. 2002;6 (6):471-479. doi:10.1053/smrv.2001.0223

20. Morin C, Gibson D, Wade J. Self-reported sleep and mood disturbance in chronic pain patients. Clin J Pain. 1998;14(4):311-314. doi:10.1097/00002508-199812000-00007

21. Edwards RR, Smith MT, Stonerock G, Haythornthwaite JA. Painrelated catastrophizing in healthy women is associated with greater temporal summation of and reduced habituation to thermal pain. Clin J Pain. 2006;22(8):730-737. doi:10.1097/01.ajp.0000210914.72794.bc

22. Deutsche Gesellschaft für Schlafforschung und Schlafmedizin. Abend/ Morgenprotokolle. Available from: http://www.dgsm.de/downloads/ fachinformationen/frageboegen/protokol.pdf. Accessed December 6, 2019.

23. Buysse DJ, Reynolds CF, Monk TH, Berman SR, Kupfer DJ. The pittsburgh sleep quality index: a new instrument for psychiatric practice and research. Psychiatry Res. 1988;28:193-213. doi:10.1016/0165-1781(89)90047-4

24. Sullivan MJ, Bishop SR, Pivik J. The pain catastrophizing scale: development and validation. Psychol Assess. 1995;7(4):524-532. doi:10.1037/1040-3590.7.4.524

25. Deutsche Schmerzgesellschaft e.V. Deutscher Schmerzfragebogen. Available from: https:/www.schmerzgesellschaft.de/schmerzfragebo gen. Accessed December 12, 2019.

26. Karmann AJ, Maihöfner C, Lautenbacher S, Sperling W, Kornhuber J, Kunz M. The role of prefrontal inhibition in regulating facial expressions of pain: a repetitive transcranial magnetic stimulation study. $J$ Pain. 2016;17(3):383-391. doi:10.1016/j.jpain.2015.12.002

27. Horn-Hofmann C, Kunz M, Madden M, Schnabel E-L, Lautenbacher S. Interactive effects of conditioned pain modulation and temporal summation of pain-the role of stimulus modality. Pain. 2018;159(12):2641-2648. doi:10.1097/j.pain.0000000000001376

28. Meyer K, Sprott H, Mannion AF. Cross-cultural adaptation, reliability, and validity of the German version of the pain catastrophizing scale. J Psychosom Res. 2008;64(5):469-478. doi:10.1016/j.jpsychores. 2007.12.004

29. Nie H, Arendt-Nielsen L, Andersen H, Graven-Nielsen T. Temporal summation of pain evoked by mechanical stimulation in deep and superficial tissue. J Pain. 2005;6(6):348-355. doi:10.1016/j.jpain.2005.01.352

30. Lautenbacher $S$, Roscher $S$, Strian F. Inhibitory effects do not depend on the subjective experience of pain during heterotopic noxious conditioning stimulation (HNCS): a contribution to the psychophysics of pain inhibition. Eur J Pain. 2002;6:365-374. doi:10.1016/S1090-3801(02) 00030-7 
31. Rechtschaffen A, Kales A. A manual of standardized terminology, techniques and scoring system for sleep stages of human subjects. Washington DC: Public Health Service, U.S. Government Printing Office; 1968.

32. O'Donoghue GM, Fox N, Heneghan C, Hurley DA. Objective and subjective assessment of sleep in chronic low back pain patients compared with healthy age and gender matched controls: a pilo study. BMC Musculoskelet Disord. 2009;10:122. doi:10.1186/14712474-10-122

33. Faul F, Erdfelder E, Lang A-G, Buchner A. G*Power 3: a flexible statistical power analysis program for the social, behavioral, and biomedical sciences. Behav Res Methods. 2007;39(2):175-191. doi:10.3758/bf03193146

34. McCracken LM, Iverson GL. Disrupted sleep patterns and daily functioning in patients with chronic pain. Pain Res Manag. 2002;7 (2):75-79. doi:10.1155/2002/579425

35. Diaz-Piedra C, Catena A, Sánchez AI, Miró E, Martínez MP, BuelaCasal G. Sleep disturbances in fibromyalgia syndrome: the role of clinical and polysomnographic variables explaining poor sleep quality in patients. Sleep Med. 2015;16(8):917-925. doi:10.1016/j. sleep.2015.03.011

36. Blågestad T, Pallesen S, Lunde LH, Sivertsen B, Nordhus IH, Grønli J. Sleep in older chronic pain patients: a comparative polysomnographic study. Clin J Pain. 2012;28(4):277-283. doi:10.1097/ AJP.0b013e3182313899

37. Finan PH, Goodin BR, Smith MT. The association of sleep and pain: an update and a path forward. J Pain. 2013;14(12):1539-1552. doi:10.1016/j.jpain.2013.08.007

38. Lavigne GJ, Okura K, Abe S, et al. Gender specificity of the slow wave sleep lost in chronic widespread musculoskeletal pain. Sleep Med. 2011;12(2):179-185. doi:10.1016/j.sleep.2010.07.015

39. Graven-Nielsen T, Kendall SA, Henriksson KG, et al. Ketamine reduces muscle pain, temporal summation, and referred pain in fibromyalgia patients. Pain. 2000;85:483-491. doi:10.1016/S03043959(99)00308-5
40. Price DD, Staud R, Robinson ME, Mauderli AP, Cannon RL, Vierck CJ. Enhanced temporal summation of second pain and its central modulation in fibromyalgia patients. Pain. 2002;99:49-59. doi:10.1016/S0304-3959(02)00053-2

41. Sörensen J, Graven-Nielsen T, Henriksson KG, Bengtsson M, Arendt-Nielsen L. Hyperexcitability in fibromyalgia. J Rheumatol. 1998;25(1):152-155.

42. Staud R, Cannon RC, Mauderli AP, Robinson ME, Price DD, Vierck CJ. Temporal summation of pain from mechanical stimulation of muscle tissue in normal controls and subjects with fibromyalgia syndrome. Pain. 2003;102(1):87-95. doi:10.1016/S0304-3959(02) 00344-5

43. Staud R, Vierck CJ, Cannon RL, Mauderli AP, Price DD. Abnormal sensitization and temporal summation of second pain (wind-up) in patients with fibromyalgia syndrome. Pain. 2001;91:165-175. doi:10.1016/S0304-3959(00)00432-2

44. Goubert D, Danneels L, Graven-Nielsen T, Descheemaeker PT, Meeus M. Differences in pain processing between patients with chronic low back pain, recurrent low back pain, and fibromyalgia. Pain Physician. 2017;20(4):307-318.

45. Gerhardt A, Eich W, Treede R-D, Tesarz J. Conditioned pain modulation in patients with nonspecific chronic back pain with chronic local pain, chronic widespread pain, and fibromyalgia. Pain. 2017;158(3):430-439. doi:10.1097/j.pain.0000000000000777

46. Horn-Hofmann C, Priebe JA, Schaller J, Görlitz R, Lautenbacher S. Lack of predictive power of trait fear and anxiety for conditioned pain modulation (CPM). Exp Brain Res. 2016;234(12):3649-3658. doi:10.1007/s00221-016-4763-9

47. Aviram J, Shochat T, Pud D. Pain perception in healthy young men is modified by time-of-day and is modality dependent. Pain Med. 2015;16:1137-1144. doi:10.1111/pme.12665

48. Plante DT, Winkelman JW. Polysomnographic features of medical and psychiatric disorders and their treatments. Sleep Med Clin. 2009;4(3):407-419. doi:10.1016/j.jsmc.2009.04.008
Journal of Pain Research

\section{Publish your work in this journal}

The Journal of Pain Research is an international, peer reviewed, open access, online journal that welcomes laboratory and clinical findings in the fields of pain research and the prevention and management of pain. Original research, reviews, symposium reports, hypothesis formation and commentaries are all considered for publication. The manuscript management system is completely online and includes a very quick and fair peer-review system, which is all easy to use. Visit http:// www.dovepress.com/testimonials.php to read real quotes from published authors. 